\title{
APPENDIX A
}

[The transcribed material on pages $\{923\}$ to $\{940\}$ consists of the entries made by Dr. John Snow as the end matter of each of his three manuscript volumes of case books. These entries are different in style and content from the majority of his other narrative entries. For clarity, they have been collated in this single section of the transcribed work. The endmatter from the first volume (originally at pages 1465 ] to [467) is on pages 1923) to \{925\}; pages \{926\} to \{938\} contain that from the second (originally at pages $\{812\}$ to \{824\}); and pages \{939\} and \{940\} that from the final volume (originally at pages \{921\} and (922)). The numbers in the right-hand column, 538-599, were Snow's own notation, the significance of which is not clear.]

Oct. 14, Fem.

Mrs. Buckingham, 6 Newport Court.

October

Oct. 29, Mas.

Mrs. Hill, ?Showfield St., Kings Road.

November

Jan. 5, Mas.

Mrs. Sutton, 3 Little St., Andrew St.

November

Oct. 16, Fem.

Mrs. Forrest, 2 St Anne's Court.

October

Nov. 2, Mas.

Mrs. Butler, 3 Great Earl St.

November

Dec. 6, Mas.

Mrs. Edwards, 21 Newport Court.

End of December

Dec. 14, Mas.

Mrs. Allen, 33 Sussex St.

January 1849

Jan. 12, Fem.

Mrs. Terry, 56 Frith St. (Mas) on Dec. 11, 1848.

Mrs. [Duhy], 23 Cecil Court.

January 1849

29 Dec. Fem.

Mrs. Barker, 40 Ampthill Square.

January

Jan. 24, Fem.

Mrs. Birt's Sister, Rose Street

February

Not attended.

Mrs. Martin, Castle St., Leicester Square.

February

Fem, Feb. 21.

Mrs. Shepherd, 14 Lumber Court.

April

Fem, 9 April.

Mrs. Leif, Crown Court, Westminster. Fem. on January 30.

Mrs. O’Donnell, 16 Little Portland St.

March

Fem, March 3.

Mrs. Metcalfe, 39 Little Pulteney St.

June

Mas, June 29. 


\section{Case Books}

Mrs. Elworthy, 8 Cecil Court.

End of May

June 12, Fem. Face Present.

Mrs. Biggs, 33 Little Earl St.

May

Fem. May 1.

Mrs. Smith, 7 Gt. White Lodge St.

June

July 14, Mas, Foot, DC.)

Mrs. Jolly, 9 Rose St. Soho.

September ? died

58

Sept. 12, Mas.

Mrs. Rowles, 4 Little Compton St.

September

Oct. 4, Mas.

Mrs. Roberts, 23 Cecil Court. Mas on Aug. 28

Mrs. Anderson, 5 Margaret St.

Dec. 11, Fem.

Mrs. Root, 8 Cecil Court.

Fem. Aug. 29.

$\begin{array}{ll}\text { Nov. } & 60 \\ & 61\end{array}$

August 62

$\{924\}$

Mrs. [Duhy], 23 Cecil Court

Nov. 16, Fem. Arm, D.C.

Mrs. Everett, 17 Harrison St., Gray's Inn Road, end of Nov.

Dec. 12, Fem.

Mrs. Poole, 17 Wardour St. Middle of October.

Oct. 13, Mas.

Mrs. Johnson, 8 Leicester St. Middle of January 1850

March 4, Fem.

Mrs. Lygo, 15 Moor Street, Soho. March

Apr. 4, Mas.

Mrs. Penry, 7 Henrietta Mews, Brunswick Square. March

March 14, Fem.

Mrs. Naylor, 17 Duke St., Lincoln's Inn Fields. March. Primip.

March 26, Mas.

Mrs. Benson, 163 New Oxford St. March. 2nd. Ch.d.

March 21, Fem.

Mrs. Goodrid, 55 Wardour St. April. 2nd. Ch.d.

April 3, Mas.

Mrs. Buckingham, Newport Court. February 1850.

March 21, Mas.

Mrs. Legasick, 23 Noel Street. May.

May 30, Mas.

Mrs. Bent, Castle St., Leicester Square. August?

574

July 22, Mas.

Mrs. Sevenoaks, 75 New Compton St. Sept.

Sept. 17, Mas.

Mrs. Smith, 2 Queen St., Golden Square. Middle of October.

Oct 1, Fem. 
John Snow

Mrs. Young, 51 Old Compton St. End of September.

19 Oct. Fem.

Mrs. Rowles, Little Compton St. November.

578

Nov. 7, Mas.

Mrs. Desmajieu, 63, King St., Westminster. December.

579

Dec. 21, Mas. D.C.

Mrs. [Duhy], Cecil Court. About Christmas.

580

Feb. 3, Fem.

581

Feb . 5, Fem.

Mrs. Smith, 5 Margaret St. Latter part of May

582

Mas. May 31.

Mrs. Elworthy, 8 Cecil Court. Beginning of May.

583

Fem. May 30.

Mrs. Biggs, 12 Moor St. Latter part of May.

584

June 7, Mas.

Mrs. Penry, 7 Henrietta Mews. Beginning of August.

585

Mas. 29 July.

Mrs. Roberts, 23 Cecil Court. Middle of September.

Mas. 7 Oct.

586

$925 \quad\{925\}$

Mrs. Root, 8 Cecil Court. Early in November.

587

Mas. Nov. 26.

Mrs. Smith, 6 Margaret Street. Middle of November.

588

Fem. Nov. 18.

Mrs. Buckingham, Newport Court. Beginning of March

589

Fem. Nov. 6.

Mrs. Everett, 46 Charrington St. November

590

Fem. Nov. 19.

Mrs. Johnson, 8 Leicester Street. January 1852.

591

Fem. Dec. 29, 1851.

Mrs. Rowles, 2 South Row. February.

592

Fem. Feb 23.

Mrs. Duren, Greek St. February.

593

Fem. Feb. 29

Mrs. Hillier, 17 Cecil Court. March 1st.

594

Feb. 6. Mas.

Mrs. Metcalfe, 39 Little Pulteney St. End of October.

595

Mas. Nov. 14.

Mrs. Desmajeux, 9 Crown Ct., Westm.r. End Of Dec.

596

Jan. 4, Fem.

Mrs. Hillier, 17 Cecil Court. March 1853.

Feb. 25, Mas.

Mrs. Rowles, 2 South Row. Mas. on Feb 231853.

Mrs. Smith, Jnr., 6 Margaret St., Cavendish Square. Middle of May. 


\section{Case Books}

$\{926\}$

Mrs Johnson 28 Coventry St., August 1853, Mas. Aug. 25th.

Mrs. Smith, 4 Richmond Buildings, End of October, Mas. on Nov. 9.

Mrs. Metcalfe, 39 Little Pulteney St., Fem. January 17, 1854.

Mrs. [Duhy], St. Ann's Court Twin Males presentation natural. Feb. 13.

Mrs. Desmajure, Crown St., Westminster, March, Mas. March 31.

Mrs. Roote, 8 Cecil Court, Middle of Feb. Fem. March 23.

Mrs. Bent, 34 Castle St. Mas. August 9.

Mrs. Barker, 39 Gloucester Road. Mas. March 19, 1855

Mrs. Roote, 8 Cecil Court, Mas. April 8, 1855.

Mrs. James Smith, 7 Great Portland Street, Mas., Nov. 18, 1855.

Mrs. Johnson, 7 Frith St. Fem. Prem on March 13, 1856.

Mrs. Metcalfe, 39 Little Pulteney St. Fem., May 6, 1856.

Mrs. Hillier, 10 Priory Grove, Gilston Road, Brompton, Mas. 6 June, 1856.

Mrs. [Duhy], 6 St. Ann's Court. === Nov. 6, 1856.

Mrs. Ladiner, 25 Adam Street East, Manchester Square.

(Amylene, Maddox St.)

$\{927\}$

May 4, 1853

Mrs. Bent Visit, \& 6, 9, 12 ,

May 6

Mr. Burbage Visit \& 7, 9, 11, 13.

May 10

Mrs. Hogarth Advice.

May 12

Mrs. Lake at Mrs. Bent's Advice 15 Visit \& 18.

May 16

Mr. Johnson, Coventry St. Visit to Child \& 18, 20, 23, 26, 29, June 1, 3, 5, 8, 12, 15, 20.

June 7

Mrs. Barker, Gloucester Road Visit \& 9th.

June 25

Mr. Johnson, Coventry St. Visit Self. 27, 29, July 1, 3. 
John Snow

June 27

Mr. Hogarth Visit 18 Two Visits 21 V. 22, V. 25, 27.

August

Mrs. Bent's Child Advice several times in Aug. and Sept.

\section{September}

Mr. Hillier Advice three or four times.

Mrs. Johnson (Coventry Street) and Child Visit 2 or 3 times a week from Sept. 23 to Oct 12. Oct. 13 Visit Child 14, 16, 18, 20, 21, Vaccinating Baby on 20th., 21 Visit 23, 25, 27 , 29, 31. Nov. $3,5,7,9,11,14,16,19$.

$928\{928\}$

October 8, 1853

Mr. Barker's Son Visit, 10, 12, 15, 20 Visit Daughter 22, 25, 28.

October 15

Miss Gray 37 Baker St., Lloyd Square visit, 20 Advice, 24 Advice, 28 Advice Nov. 2, 6, 10 Consultation at Dr. Billing's, 14 Advice.

Mrs. Williamson Visit on Nov 18, 19, 20, 21, 23, Two Visits 24.

Nov. 18

Miss Gray Visit 22 Advice, 26, Dec. 2, 8, Visit 16 Visit 20, V.

\section{November 23}

Mrs. Johnson, Coventry St. Visit and 24, 25, 26, 27, 28, 29, 30 Dec. 1, 3, 6, 8, 11, 12, 14.

Dec. 4

Mrs. Hillier's Baby Visit 5, 6, 8, 11,

Dec. 8

Mrs. Irving Visit, 11, 13, 15, 18, 21, 24, 27.

Dec. 15

Mrs. Johnson's Child Visit, 16, 17, 19, 21.

Dec. 26

Miss Gray Visit. 


\section{Case Books}

January 2, 1854

Miss Gray Visit, 6, 12, 16, 20, 25, Feb. 1, 7, 14, 21, 28 Advice.

January 9, 1854

Mrs. Irving Visit, 11 \& Mr. 13, 15, 18, 20, 22, 29, Feb. 5, 12, 13, 14, 16, 18, 21, 24, 27.

January 20

Mrs. Anderson, 30 Margaret St. Visit, 23, 25, 27, Feb 8, 11,

January 24

Mrs. Barker's Son Louis Visit.

January 28

Mrs. James Smith's Infant, 6 Margaret St. Reducing fractured clavicle.

Mrs. Smith's Infant, 4 Richmond Buildings. Vaccination January 31.

February 13

Mrs. [Duhy]. Delivery Twins, Males, Vertex and Breech.

February 23

Mr. Roote Visit, 27, March 1, 5.

March 3

Mr. Irving Visit, 7.

March 8

Miss Gray Visit 15 Advice, 23 Visit March 30 Advice Apr. 7 Ad 19.

March 24

Mrs. Roote, Delivery (Fem).

March 27

Mr. W. Smith's Son, 6 Margaret St. Visit, 28, 29, 31, Apr. 2, 4, 5, 6, 7.

April 8

Mr. W. Smith's Children Visit. 9, 10, 11, 12, 13, 15, 20. 


\section{John Snow}

\section{April 16}

Mr. Anderson, 30 Margaret St. Advice 18, Visit May 1, Advice 21.

\section{April 22}

Mr. Hogarth Advice Son John, 25, 26, 28, 30, May 2, 4, 6, 8, 10, 12, 14, 16, 17, 18.

\section{April}

Mrs. Bent's 2nd. Child Advice May 3 Advising Eldest 6 V. 7 Visit, 8, 9, 10, 11, 12, 14, 16, 18.

$930 \quad\{930\}$

May 2, 1854

Miss Gray. Advice 11, 18, 25, June 1, 8, 17.

\section{May 8}

Mr. Smith's Children, 4 Richmond Buildings Visit, 9, 10, 11, 12, 14, 16, 18.

\section{May 9}

Mrs. Hogarth Three V. 10, Two V. 11, 12, 13, Advice, 16.

\section{May 12}

Mrs. Barker, 37 Gloucester Road Advice, 14, Visit 18.

\section{May 19}

Mr. John Hogarth Advice, 20, 21, 22, 23, 24, 25, 26, 27, 29, 31, June 2, 4, 6, 8, 10, 12.

May 19

Mrs. Bent's Eldest Child Visit 20, 21, 22, 23, 24, 25, 26, 27, 28, 29, 30, 31.

June 1st.

Mr. Bent's Eldest Child Visit 2, 3, 4, 5, 6, 7, 8, 9, 10, 12, 13, 14, 15, 16, 17, 18, 19, 20, 21.

June 1st.

Mrs. Roote's Infant. Vaccination.

June 22nd.

Mr. Bent's Child. Visit daily till August 7th. 


\section{Case Books}

July

Miss Gray Advice 3 or 4 times by letter to Tonbridge.

August 1st.

Mrs. Ripley Visit 2, 4, 5, 6, 8, 10.

August 4

Mrs. Gray Visit 12, Advice and 2 or 3 more times till Sept. Oct. 28 Advice 30 Visit.

August 7

Mrs. Roote. Visit in the night. Visit 8, V. 9, V. 22, Visit Infant 23, 24.

August 9

Mrs. Bent. Delivery (Mas).

August 10

Mrs. Barker, 37 Gloucester Road, Visit, 15 V.

Sept. 10

Mrs. Irving Visit 11, 13.

\section{Sept. 1}

Mr. Bent's Child, about 10 visits to the 15 th.

\section{Sept. 24}

Mr. Bent 26, 27, 28, 30. Oct. 10, 11, 12, 14, 16, 18.

Oct. 10

Mr. T. J. Barker Visit, 12.

Oct. 13

Mr. Irving Visit to Mr. Danskin at Sydenham.

15. Visit to Mr. D. at Mr. Irving's 16, 17, 18, 20, 22, Mrs. Irving 26, 30 Self.

Oct. 26

Mrs. Bent's Infant. Vaccination. Attendance Edward twice or thrice a week to Dec. 31 st. 


\section{John Snow}

Nov. 2

Miss Gray Visit 9.

Nov. 11

Mrs. Irving. Visit to Mr. Danskin, 13 Visit to Mrs. Irving.

Dec. 15

Mrs. Hillier Visit, 16 Visit 18, 20, 23.

$932\{932\}$

January 5, 1855

Mr. Hogarth. Visit, 6, 7, \& George 8, 9, 10, 11, 12, 13, Geo. 15, 17, 19, 20, 21, 22, 23, 26, 28, 31, Feb. 3, 6, 10, 17.

Mrs. Hillier's Baby. Visit January 8, 9, 10, 11, 12, 14, 17, 20, 27.

\section{January 9}

Miss Gray Advice. Mr. George twice in Jan? \& on Feb 5.

January 11

Mr. Wills, 24 Northumberland St. 2 Visits \& consultation with Mr. Marshall. 12 Visit \& consultation.

January 17

Mr. Irving. Visit.

January 24

Mrs. Smith, 4 Richmond Buildings. Visit \&c. 25, 26, 28, 30, Feb. 2.5.9.14.

January 26

Mrs. James Smith's Baby Visit, 28, 30, Feb 2.

Feb. 9

Mr. \& Mrs, Gray Visit, 26 Mr. Gray Advice March 5, 6 at Dr. Billing's, 8 Miss 16 Mr.

Feb. 9

Mrs. Barker Visit 11, March 14, 19 Delivery, 20 V, 21, 22, 23, 25, 27, 30, Apr. 3, 9,

March 27

Miss Gray. Visit. Apr. 3. Mr. G. Advice, 12, 17, Miss, 23, Mr. 26 V. Miss. 


\section{Case Books}

\section{April 8}

Mr. Smith's Child, 4 Richmond Buildings 2 Visits, 9.2 Vs., 10 2V., 11 2V, 12 3V, 13 2V, $142 \mathrm{~V}, 153 \mathrm{~V}, 162,172 \mathrm{~V}, 182 \mathrm{~V}, 192 \mathrm{~V}$.

\section{April 8}

Mrs. Roote, Delivery (Mas).

$\{933\}$

April 20

Mrs. Barker. Visit 21, 23.

\section{April 28}

Mrs. Irving. V. 30, May 2, 3, 4, 5, 6, 6, 7, 8, 9, 10, 11, 12, 14, 16, 19, 21, 23, 25, 26, 28, 31.

\section{April 30}

Mrs. Johnson, 20 Gerrard St. V. May 1, 2, 3, 3, 4, 4, 5, 5, 6, 6, 7, 8, 10, 17.

\section{May 1}

Mrs. Ripley. V. 2, 4, 7, 10, 15, 25.

\section{May 11}

Miss Gray. V. 15, V. 19, Advice 21, Mr. 25 V. Mr. \& Miss June 4th. Advice.

Mr. 14th, 21st.

\section{May 20}

Mr. Hogarth. Visit, 31 V. June 1st., 2, 3, 5, 13. Andrew 14, 16.

May 19

Mrs. Allen's Son, Henry, 17 Percy St. V. 20 V.

May 28

Mr. Barker's Son, Louis V. 29th., 31st. Vac. Infant.

June 1st.

Mrs. Irving V. 3rd., 5th., 7, 9, 11, 13, 16. June 27, 28, 30, July 2, 4, 6, 9, 14, 18, 23.

Aug. 29

Mrs. Johnson, 39, 31, June 1, 2, 4, 6, 7, 8, 9, 11, 13, 16, June 27, 28, 30, July 2, 4, 6, 9, 14 , 18, 23. 


\section{John Snow}

June 1st.

Mrs. Roote's Infant. V. 2nd., 5, 21 st. Vac.

June 23

Miss Hogarth. V. 24, 25, 27, 29, 30. July 2, 5, 14 Advice Sept. 17.

June 26

Miss Gertrude Wallace. V. at Canonbury Place, 28. £1.1.0.

July 6, 1855

Mrs. Wallace. Visit to Miss Wallace at 122 Warwick St., 9, 10, 11, 13, 15, 18, 21, 25. £3.3.0.

July 17

Mrs. Irving. Advice 19, Aug. 6, 7, 9, 10, 12, 13, 14, 15 V., 17 18, 20.

Aug. 13

Mr. Anderson, 30 Margaret St. Visit, 14, 15, 18, 19, 20, 21, 22, 23, 24, 26, 28, 30, 31.

When I was called to Mr. Anderson he had been bleeding from the nose, but he had stopped it by the application of turpentine on cotton before I arrived. He had large patches of purpura haemorrhagica on his arms, hands, thighs and legs, and some smaller ones on his head. They had come out at various times the three previous days, some of the earlier ones were already fading. There was, in the centre of each patch of purpura, a small hard swelling or nodule, over which the skin was quite white. These nodules felt as hard almost as cartilage, and were proportionate in size to the patches of purpura. Where these were as big as the hand, the nodules were as big as a horse bean; where the patches were smaller so were the nodules. It appeared to me that the nodules probably consisted of fibrin, that the blood had been effused at these points and the fibrin had become congelated whilst the rest of the blood had spread in the skin and under the cuticle. He had been drinking very hard for some time, and eating scarcely anything. His limbs were rather tremulous, pulse bounding but not strong. To take lemon juice, and a tonic with diluted sulphuric acid. He improved during the next three days, the patches faded, and the nodules diminished in

935 size. $\{935\}$ When called to him on the 18th. I found him to be labouring under erysipelas of the face and head. There had been a little swelling and redness of the right ear on the evening of the 15th. which had since extended. To take Quinine and apply solution of nitrate of silver to the face. The hairy scalp was not so much affected as the face \& forehead. (25th. Has gradually improved, and face nearly well.) On the 23rd. he was making very little urine, only two or three ounces in the morning after lying all night. It was clear and of straw colour, sp. gr. 1.015, not albuminous. Feels low. P. 84, not feverish. Has had no fermented liquor since his illness. 30th. A return of the erysipelas in the face 


\section{Case Books}

after going out and eating very freely at supper. It did not become very bad, and was subsiding on Sept. 3rd.

\section{August 27th.}

Miss Gray. Advice Sept. 10 Oct. 6 V. Advice 25. Ad. Mrs. G. 30 V. Miss.

\section{August 29th.}

Mr. Chas. Hillier. V. 31, Sept. 2, 4, Advice 20.

\section{August 29th.}

Mr. Dearer's Son. V., 30, 31, Sept. 1, 2, 3, 4, 5, 7, 8, 10, 13, 19.

\section{August 30th.}

Mrs. Johnson. V. 31, Sept. 1, 3, 5, 7, 10, 11, 12, 13, 14, 21.

Sept. 5th.

Mr. Hogarth. V. 7, 8, 12, 13.

Sept. 6th.

Mr. I. J. Barker. V. 8.

$\{936\}$

Sept. 26, 1855

Mrs. Irving. V. and Leeching. Oct. 4. Advice. 21, 25, 30, Nov. 11, Dec 8, V.

October 20

Mrs. Hillier. Visit. 21, 23.

Nov. 1

Mrs. Roote. Visit. 2, 4, 6, 9. Infant Dec. 3, 5, 7.

Nov. 10

Miss Gray. V. 15, V. 22, 29 Advice.

Nov. 28

Mrs. Hogarth. V. 30, Dec. 4, 7, 11.

Dec. 8

Mr. Barker. Advice 15. 


\section{John Snow}

January 7, 1856

Miss Gray. Visit, 20, 23 Advice, \&c. Feb. 9 Ad. Miss 16 V. Mr. 23 Ad.

January 10

Mr. Hogarth. Advice.

January 17

Mrs. T. J. Barker. V. 19. Feb. 24. Mr. and Sophie 27. March 1, 4, 6, 8. Mr. \& Louis.

January 15

Mrs. Ripley. Advice, 19, V. 25 Ad.

January 24

Mrs. Johnson, 7 Frith St. V. 25, 26, 27, 28, 31, Feb. 1, 3, 4, 5, 7, 10, 11, 13, 15, 17, 19, 22 , 25.

\section{February 7}

Mrs. Hillier. Advice. March 8, Child Ad.

$937 \quad\{937\}$

February 19

Mrs. Hogarth. Two Visits. 20, 21, 22, 23, 25, 27, 29, March 3, 5 and April 6, 8, 11, 14, 17 , 20.

\section{March 7}

Mr. Johnson, 7 Frith St. V. 9, 11, 13. Delivery Fem.

March 12

Mr. Allen, Percy St. V. 14, 17, 22 Advice.

March 11

Mrs. Roote's Infant. V. 12, 15, 17.

\section{March 22}

Mrs. Hogarth. 2 Visits 23, 25.

\section{March 18}

Mr. Grey. V. to Dr. Billing. Apr 3. Miss G. Ad. 5. Mr. G. Ad. 8, May 14. Miss G. Ad. 17 Visit. 


\section{Case Books}

May 23

Mrs. Barker's Infant, Gloucester Road, Visit 25.

May 30

Mr. Irving. V. June 1, 3, 4, 6, 8, 10, 12, 14, 16, 18, 21, 26.

June 6

Mrs. Hogarth 2 Visits, 7. V.

June 6

Mrs. Hillier, 10 Priory Grove, Brompton. Delivery Mas.

July 1

£2-14-0 Paid.

Mr. Smith's Child, 83 King St., Camden Town. 2, 3, 4, 6, 8, 9, 11, 14, 16, 18, 20, 22, 24, 26, 28, 30, Aug. 2.

\section{August 13}

Mr. Hogarth. V. Oct. 14 Advice 17, 21.

$\{938\}$

Oct. 14, 1856

Miss Gray. Advice.

Nov. 20

Mrs. Hogarth. Visit. Dec. 1, 9.

Nov. 30

Mrs. Irving. Advice. Dec 8th. Visit.

Dec. 28

Mr. I. J. Barker. Visit to Louis. 29th. 30th.

Jan'y. 1st., 1857

Mr. I. J. Barker. Visit to Louis. 3rd., 6th. Visit other children 7th., 9, 10.11, 12, 14, 18, 23.

Jan'y. 25, 1857

Miss Gray. Visit at St. Paul's Villas, Paul's Road, Camden Town. Feb 3, 5, 7, 10, 13, 16, 20, 24, 26, 28, Mar. 2, 5, 8, 12. 


\section{John Snow}

Feb. 9

Mrs. Hogarth. Visit. 11, 15, 20, Mar. 4, 5, 6, 10.

Feb. 7

Royal Oak Benefit Society. Examining John Hall.

\section{March 16}

Miss Gray. Visit. 21, 25, 28, Apr. 4, 10, 17, 24, May 1, 8, 16.

June 9

Mr. Hogarth's Son, George. V. 10, V.V. 11, V. 12, V. 14, V.

$939\{939\}$

July 20, 1857

Mrs. Hillier, Brompton. Visit. 10/6.

July 24

Mrs. Irving. Advice.

Aug. 4th.

Mrs. Hogarth's Son, Geo. Admin. Chloroform 5 V, V, 6 V, 7, 8, 10.

\section{Sept 21}

Miss Gray Visit Nov. 4, 8, 11, 16, 22, 24, Dec 4, 22.

Oct. 14

Mrs. Barker's Baby Visit.

Nov. 1

Mrs. Hogarth's Son Charles Visit 2, 3, 4, 5, 6, 7, 8, 9, 10, 11, 12th. 3 Visits 13th. 3 V. 14th. $3 \mathrm{~V}$.

Mr. Hogarth's Son Charles 3 Visits Nov 15, 3 V. Nov. 16.

Dec. 16

Mr. I. James Barker Visit. 


\section{Case Books}

1858

Jan'y. 1st. Friday

Mrs. Hogarth V. 3, 4, 5, 7, 10, 13, 17.

Feb. 16

Mr. John Hogarth V. 18, 20, 22, 24, 26, 28, March 2, 4, 6, 8, 9, 10, 12, 14, $17,20$.

Feb. 17

Miss Gray Visit.

March 5

Mr. I. J. Barker Visit April 12, Visit Baby 14, 16, 18, 20, 22, 24.

$\{940\}$

25 Adam Street East, Manchester Square. 